\title{
Director profile of a nematic between two concentric cylinders with inhomogeneous boundary conditions
}

\author{
C.A.R. Yednak ${ }^{1,2}$, E.K. Lenzi ${ }^{1,2},{ }^{*}$ and L.R. Evangelista ${ }^{1}$ \\ ${ }^{1}$ Departamento de Física, Universidade Estadual de Maringá Avenida Colombo, 5790-87020-900 Maringá (PR), Brazil and \\ ${ }^{2}$ Dipartimento di Fisica del Politecnico di Torino, Corso Duca degli Abruzzi 24, 10129, Torino Italia
}

(Received on 10 February, 2009)

\begin{abstract}
The tilt angle profile in a nematic cell limited by two concentric cylindrical surfaces with inhomogeneous distribution of easy axes is investigated in the one-constant approximation. The results are presented in terms of the Green function approach by considering the strong anchoring case and the presence of an external electric field for small distortions.
\end{abstract}

Keywords: Liquid crystal; Nematic; Strong anchoring

\section{INTRODUCTION}

Systems that involve liquid crystal alignment between two concentric cylinders have been investigated in connection with the flexoelectric instability [1], with the stability analysis of the orientational profile [2], and the Fréedericksz transition occurring in the absence of external electric field [3]. The starting point of these analysis was the original problem proposed by Meyer, solved in a special case by Parodi, and then discussed in the book by de Gennes [4]. Subsequently, the same problem was reexamined by Williams [5] by considering that the elastic constants of splay and bend are different, in the strong anchoring approximation. Very recently, the equilibrium problem for a nematic liquid crystal confined within two parallel eccentric cylinders with homeotropic anchoring on the lateral boundaries has been rigorously analyzed by Rosso et al. [6] in the framework of a purely director approach. In general, problems dealing with the equilibrium orientational states of nematic liquid crystals are faced in the framework of the elastic continuum theory for liquid crystalline materials. The basic principle of this theory is that the distorted state can be described by the director $\mathbf{n}$ that is of unit length but can be of variable orientation, and represents the average molecular orientation. The equilibrium configuration is the one minimizing the total free energy of the sample subjected to appropriate boundary conditions [7, 8]. The determination of the equilibrium director profile can be formulated in terms of boundary value problems [9]. The boundary value problem concerning the situation of strong anchoring on the boundaries corresponds to the Dirichlet's problem, whereas the weak anchoring situation leads to the mixed Dirichlet - Neumann problem. On the other hand, the situation in which the easy axes characterizing the preferred surface alignment change direction continuously with time is relevant to investigate systems whose surfaces are covered with photopolymeric films [10]. In these systems, the orientational changes of the photochromic molecules promoted by incident light lead to remarkable changes in the molecular orientation. In this direction, problems dealing with orientational dynamics and surface viscosity have also been investigated by Mertely and Copic [11]. For this reason, in order to account for these characteristics of a nematic sam-

*Electronic address: eklenzi@dfi.uem.br ple confined between two cylinders, we face here a generalization of the model treated in Refs. [3,5] by considering a system formed by inhomogeneous surfaces, i.e., by considering that on each cylindrical surfaces the treatment has ensured a spatial distribution of the easy directions. Furthermore, the present approach incorporates a time dependence in the distribution of easy axes on the surfaces, in the situation of strong anchoring, which is a more difficult mathematical problem, but can represent a more realistic physical situation. In this manner, our analysis is quite general in the framework of the usual approximation of one-elastic constant; it takes into account an external field, directed along the radius of the cylinders, in the limit of small distortions. To face the dynamical reorientation problem in a complete manner it is necessary to take into account that the motion of the fluid is coupled with the fluid flow, i.e., to consider backflow effects. Thus, besides playing an important role in the response of a nematic liquid crystal to an applied field, the molecular reorientation can generate a flow which in turn affect the reorientation $[12,13]$. As underlined by Svensek and Zumer, there has been little or no work done in the backflow problems in severely confined geometry like the one we are considering in this work. This happens because, in this case, backflow is a consequence of the confinement. Therefore, the resulting flow patterns are quite complicated and even keeping the small distortion approximation as we are doing in the present analysis, the set of equations to be solved are hardly treatable in analytical manner [14]. For this reason, we restrict the present analysis to the very simplified case in which backflow effects are not taken into account. Anyway, the formalism presented here in terms of Green function constitutes the appropriate mathematical framework to explore orientational field effects in nematic samples in confined geometries and can be extended to the situation of weak anchoring on the boundaries without significant difficulty.

\section{THE MODEL}

Let us consider a nematic liquid crystal cell limited by two concentric cylinders of radius $a$ and $b>a$, whose cylindrical reference frame is such that the $z$ axis is parallel to the cylinder axes, as shown in Fig. 1. For this geometry, the director can be written as $\mathbf{n}=\cos \psi(\rho, \theta, t) e_{\rho}+\sin \psi(\rho, \theta, t) e_{\theta}$, where the cylindrical unit vectors are related to the Cartesian unit vectors by $e_{\rho}=\cos \theta \mathbf{i}+\sin \theta \mathbf{j}, e_{\theta}=-\sin \theta \mathbf{i}+\cos \theta \mathbf{j}$, 


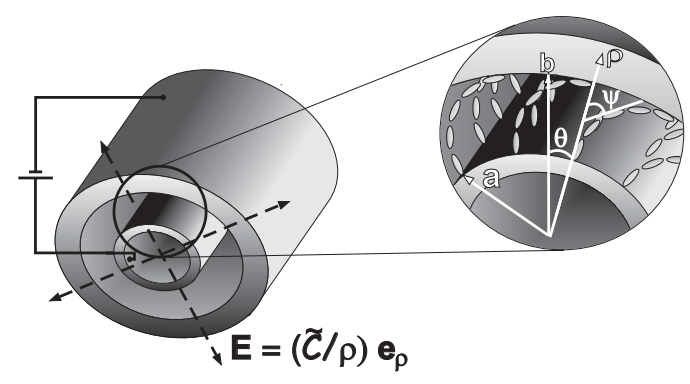

FIG. 1: Nematic sample limited by two concentric cylinders of radius $a$ and $b$. A uniform electric field is directed along a radial direction (normal to the cylinder axes, $e_{z}$ ). The director angle $\psi$ is also shown for a hypothetical distortion in the plane.

and $e_{z}=\mathbf{k}$. To make the problem analytically treatable, we assume small distortions and only splay-bend deformations. In this manner, our approach is valid for an applied field that is near the Fréedericksz threshold field. Notice, however, that the Fréedericksz transition in this system is actually a Fréedericksz-like transition when the starting configuration is a pure splay conformation, with a radial director. In the presence of a radial electric field, for a nematic liquid crys- tal with a negative dielectric anisotropy $\left(\varepsilon_{a}=\varepsilon_{\|}-\varepsilon_{\perp}<0\right)$, where $\|$ and $\perp$ refer to the direction of $\mathbf{n}$, a bend distortion can be found in the system only when the orientation on the boundaries is homeotropic [3]. On the contrary, for strong planar anchoring on the boundaries, a distortion can be found only for $\varepsilon_{a}>0$. Furthermore, a sample like the one we consider could be prepared by firstly applying a very strong uniform field along the cylinder axes in order to achieve a homeotropic uniform alignment in a plane perpendicular to the axes of the cylinders. After that, an electric field in the radial direction should be considered. The electric field considered here appears when the surfaces of the sample, located at $\rho=a$ and $\rho=b$, are subjected to a constant potential difference. A typical situation is obtained when the surface $\rho=a$ is subjected to an electric potential $\varphi=V / 2$ and the surface $\rho=b$ to an electric potential $\varphi=-V / 2$. For this case, after solving the Maxwell equations, one obtains that $\mathbf{E}=(\widetilde{\mathcal{C}} / \rho) e_{\rho}$ with $\widetilde{\mathcal{C}}=V / \ln (b / a)$. In this manner, in the one-constant approximation, i.e., $K_{11}=K_{22}=K_{33}=K$, and by taking the external field $\mathbf{E}=(\widetilde{\mathcal{C}} / \rho) e_{\rho}$ into account, the total elastic free energy per unit length along the $z$ axis is given by

$$
\begin{aligned}
F[\psi(\rho, \theta, t)] & =\int_{0}^{2 \pi} d \theta \int_{a}^{b} \rho d \rho\left[\frac{1}{2} K(\nabla \psi(\rho, \theta, t))^{2}+\frac{1}{2 \rho^{2}} \varepsilon_{a} \widetilde{C}^{2} \psi^{2}(\rho, \theta, t)\right] \\
& +\int_{0}^{2 \pi} d \theta \int_{a}^{b} \rho d \rho\left[\frac{1}{\rho^{2}}\left(2 \frac{\partial}{\partial \theta} \psi(\rho, \theta, t)+1\right)\right]
\end{aligned}
$$

To analyze the dynamics of the orientation induced by the field we have to consider also a viscous torque. By minimizing Eq. (1), taking into account a viscous torque, we find that the dynamical evolution of the system is governed by the equation

$$
\frac{\partial}{\partial \widetilde{t}} \psi(r, \theta, \widetilde{t})=\nabla^{2} \psi(r, \theta, \widetilde{t})-\frac{\gamma^{2}}{r^{2}} \psi(r, \theta, \widetilde{t}),
$$

written in a non-dimensional form by introducing a reduced coordindate $r=\rho /(b-a)$, and a reduced time $\widetilde{t}=t / \tau_{v}$, where $\tau_{v}=\lambda(b-a)^{2} / K$ is a viscous relaxation time for a sample in the shape of a slab of thickness $b-a$, when $\lambda$ is an effective viscosity coefficient of the liquid crystal $[15,16]$. Furthermore, in Eq. (2), $\gamma^{2}=\pi^{2}\left(E_{0} / E_{c}\right)^{2}$, where $E_{0}=\widetilde{C} /(b-a)$ and $E_{c}^{2}=\pi^{2} K / \varepsilon_{a}(b-a)^{2}$ corresponds to the threshold field for the Fréedericksz transition in a sample of slab shape whose thickness is $b-a$, in the strong anchoring situation at the surfaces [7]. The last term in (1) does not enter in the Euler-Lagrange equation which contains the elastic term only through the Laplace-operator acting on $\psi$. This equation needs to be solved subjected to the boundary conditions $\psi(\alpha, \theta, \tilde{t})=\Phi_{a}(\theta, \widetilde{t})$ and $\psi(\beta, \theta, \widetilde{t})=\Phi_{b}(\theta, \widetilde{t})$ which are relevant to the strong anchoring situations, where, for simplicity, non-dimensional quantities $\alpha=a /(b-a)$ and $\beta=b /(b-a)$ have been introduced. Notice that these boundary conditions account for inhomogeneous surfaces, i.e., the distribution of easy directions is spatially dependent. Furthermore, we have also generalized the calculation to incorporate a time dependent distribution of easy axes. As pointed out above, a physical situation in which a time-dependent easy direction can be achieved can be found in systems formed by photochromic molecules that, under illumination, can undergo substantial change in their orientation, and, in turn, can produce a time dependent easy direction. The initial condition, i.e., how the system was initially prepared, is $\psi(r, \theta, 0)=\psi_{0}(r, \theta)$. In order to analyze this problem, we first consider the absence of electric field and after we incorporate this field in our calculation. In absence of electric field, Eq. (2) can be written as

$$
\frac{\partial}{\partial \widetilde{t}} \psi(r, \theta, t)=\nabla^{2} \psi(r, \theta, t)
$$

with $\psi(\alpha \mid \beta, \theta, \widetilde{t})=\Phi_{a \mid b}(\theta, \widetilde{t})$ and $\psi(r, \theta, 0)=\psi_{0}(r, \theta)$. The Green function approach [17] gives the solution for Eq. (3) as follows: 


$$
\begin{aligned}
\psi(r, \theta, \widetilde{t}) & =-\int_{0}^{2 \pi} d \theta^{\prime} \int_{\alpha}^{\beta} d r^{\prime} r^{\prime} \mathcal{G}\left(r, \theta, \widetilde{t} ; r^{\prime}, \theta^{\prime}, 0\right) \bar{\psi}_{0}\left(r^{\prime}, \theta^{\prime}\right) \\
& +\int_{0}^{\widetilde{t}} d t^{\prime} \int_{0}^{2 \pi} d \theta^{\prime}\left[\left.\beta \bar{\psi}\left(\beta, \theta^{\prime}, t^{\prime}\right) \frac{\partial}{\partial r^{\prime}} \mathcal{G}\right|_{r^{\prime}=\beta}-\left.\alpha \bar{\psi}\left(\alpha, \theta^{\prime}, t^{\prime}\right) \frac{\partial}{\partial r^{\prime}} \mathcal{G}\right|_{r^{\prime}=\alpha}\right],
\end{aligned}
$$

where $\mathcal{G}=\mathcal{G}\left(r, \theta, \widetilde{t} ; r^{\prime}, \theta^{\prime}, t^{\prime}\right)$ is obtained by solving

$$
\begin{aligned}
& \nabla^{2} \mathcal{G}\left(r, \theta, \widetilde{t} ; r^{\prime}, \theta^{\prime}, t^{\prime}\right)-\frac{\partial}{\partial \widetilde{t}} \mathcal{G}\left(r, \theta, \widetilde{t} ; r^{\prime}, \theta^{\prime}, t^{\prime}\right) \\
& =\frac{1}{r} \delta\left(r-r^{\prime}\right) \delta\left(\theta-\theta^{\prime}\right) \delta\left(\widetilde{t}-t^{\prime}\right),
\end{aligned}
$$

with

$$
\begin{aligned}
& \mathcal{G}\left(\alpha, \theta, \widetilde{t} ; r^{\prime}, \theta^{\prime}, t^{\prime}\right) \\
& =\mathcal{G}\left(\beta, \theta, \widetilde{t} ; r^{\prime}, \theta^{\prime}, t^{\prime}\right)=0 \quad \text { and } \quad \mathcal{G}\left(r, \theta, \widetilde{t} ; r^{\prime}, \theta^{\prime}, t^{\prime}\right)=0,
\end{aligned}
$$

for $\tilde{t}<t^{\prime}$. The Green function which satisfies Eq. (5) may be obtained by using the eigenfunctions of the Sturm-Liouville problem related to the spatial operator. Thus, we obtain that

$$
\begin{aligned}
\mathcal{G}\left(r, \theta, \widetilde{t} ; r^{\prime}, \theta^{\prime}, t^{\prime}\right) & =-\frac{\pi}{4} \sum_{n=1}^{\infty} \mathcal{N}_{0 n} \Psi_{0 n}\left(r, k_{0 n}\right) \Psi_{0 n}\left(r^{\prime}, k_{0 n}\right) e^{-k_{0 n}^{2}\left(\tilde{t}-t^{\prime}\right)} \\
& -\frac{\pi}{2} \sum_{m=1}^{\infty} \sum_{n=1}^{\infty} \mathcal{N}_{m n} \Psi_{m n}\left(r, k_{m n}\right) \Psi_{m n}\left(r^{\prime}, k_{m n}\right) \cos \left(m\left(\theta-\theta^{\prime}\right)\right) e^{-k_{m n}^{2}\left(t-t^{\prime}\right)}
\end{aligned}
$$

with

$$
\begin{aligned}
\Psi_{m n}\left(r, k_{m n}\right) & =\mathrm{J}_{m}\left(k_{m n} r\right) \mathrm{N}_{m}\left(k_{m n} \alpha\right)-\mathrm{J}\left(k_{m n} \alpha\right) \mathrm{N}_{m}\left(k_{m n} r\right) \\
\mathcal{N}_{m n} & =\frac{k_{m n}^{2}}{\left[\left(\mathbf{J}_{m}\left(k_{m n} \alpha\right) / \mathbf{J}_{m}\left(k_{m n} \beta\right)\right)^{2}-1\right]},
\end{aligned}
$$

where $\mathbf{J}_{m}(x)$ and $\mathrm{N}_{m}(x)$ are the Bessel functions of first and second species, and $k_{m n}$ are solutions of the eigenvalue equation

$$
\mathbf{J}_{m}\left(k_{m n} \beta\right) \mathbf{N}_{m}\left(k_{m n} \alpha\right)-\mathbf{J}_{m}\left(k_{m n} \alpha\right) \mathbf{N}_{m}\left(k_{m n} \beta\right)=0 .
$$

The first term of Eq. (4) gives the dynamical evolution of the initial configuration of the system and the second term represents the "surface effect" on the first term. In particular, if the system, depending on the choice of the external field and the boundary conditions, presents a stationary state, it is manifested by the second term of Eq. (4). In this manner, Eq. (4) shows how the surface plays an important role on the time dependent behavior of the director angle of this system. This result found for the $\psi(r, \theta, \widetilde{t})$ in a confined region, i.e., $\alpha \leq r \leq \beta$, may be extended to a semi-infinite region, i.e., $\alpha \leq r<\infty$. For this case, it becomes

$$
\begin{gathered}
\psi(r, \theta, \widetilde{t})=-\int_{0}^{2 \pi} d \theta^{\prime} \int_{\alpha}^{\infty} d r^{\prime} r^{\prime} \widetilde{\mathcal{G}}\left(r, \theta, \widetilde{t} ; r^{\prime}, \theta^{\prime}, 0\right) \psi_{0}\left(r^{\prime}, \theta^{\prime}\right) \\
-\left.\int_{0}^{\widetilde{t}} d t^{\prime} \int_{0}^{2 \pi} d \theta^{\prime} \alpha \Phi_{a}\left(\theta^{\prime}, t^{\prime}\right) \frac{\partial}{\partial r^{\prime}} \widetilde{\mathcal{G}}\left(r, \theta, \widetilde{t} ; r^{\prime}, \theta^{\prime}, t^{\prime}\right)\right|_{r^{\prime}=\alpha}, \quad(9)
\end{gathered}
$$

where

$$
\begin{aligned}
\widetilde{\mathcal{G}}\left(r, \theta, \widetilde{t} ; r^{\prime}, \theta^{\prime}, t^{\prime}\right) & =-\frac{1}{2 \pi} \int_{0}^{\infty} d k k e^{-k^{2}\left(t-t^{\prime}\right)} \frac{\Psi(r, k) \Psi\left(r^{\prime}, k\right)}{\mathrm{J}_{0}^{2}(k \alpha)+\mathrm{N}_{0}^{2}(k \alpha)} \\
& -\frac{1}{\pi} \sum_{m=1}^{\infty} \cos \left(m\left(\theta-\theta^{\prime}\right)\right) \int_{0}^{\infty} d k k e^{-k^{2}\left(t-t^{\prime}\right)} \frac{\Psi(r, k) \Psi\left(r^{\prime}, k\right)}{\mathrm{J}_{m}^{2}(k \alpha)+\mathrm{N}_{m}^{2}(k \alpha)}
\end{aligned}
$$

It should be emphasized that while this limiting procedure poses no mathematical problem, it can lead to a physical sit- uation that is not very meaningful because mechanical in- 
stabilities should make the director escape out of the $e_{\rho}, e_{\theta}$ plane [18, 19].

Now, let us incorporate in our analysis the electric field, $\mathbf{E}=(\widetilde{\mathcal{C}} / \rho) e_{\rho}$. Even in this more difficult situation, we can obtain the exact solution for the profile of the tilt angle. In this scenario, Eq. (2) may also be analyzed by using the Green function approach as in the previous case and has as solution Eq. (4), with the Green function given by

$$
\begin{aligned}
\mathcal{G}\left(r, \theta, \widetilde{t} ; r^{\prime}, \theta^{\prime}, t^{\prime}\right) & =-\frac{\pi}{4} \sum_{n=1}^{\infty} \widetilde{\mathcal{N}}_{0 n} \widetilde{\Psi}_{0 n}\left(r, k_{0 n}\right) \widetilde{\Psi}_{0 n}\left(r^{\prime}, k_{0 n}\right) e^{-k_{0 n}^{2}\left(\tilde{t}-t^{\prime}\right)} \\
& -\frac{\pi}{2} \sum_{m=1}^{\infty} \sum_{n=1}^{\infty} \widetilde{\mathcal{N}}_{m n} \widetilde{\Psi}_{m n}\left(r, k_{m n}\right) \widetilde{\Psi}_{m n}\left(r^{\prime}, k_{m n}\right) \cos \left(m\left(\theta-\theta^{\prime}\right)\right) e^{-k_{m n}^{2}\left(\tilde{t}-t^{\prime}\right)}
\end{aligned}
$$

with

$$
\begin{aligned}
\widetilde{\Psi}_{m n}\left(r, k_{m n}\right) & =\mathrm{J}_{\xi_{m}}\left(k_{m n} r\right) \mathrm{N}_{\xi_{m}}\left(k_{m n} \alpha\right)-\mathrm{J}_{\xi_{m}}\left(k_{m n} \alpha\right) \mathrm{N}_{\xi_{m}}\left(k_{m n} r\right) \quad \text { and } \\
\widetilde{\mathcal{N}}_{m n} & =\frac{k_{m n}^{2}}{\left[\left(\mathrm{~J}_{\xi_{m}}\left(k_{m n} \alpha\right) / \mathrm{J}_{\xi_{m}}\left(k_{m n} \beta\right)\right)^{2}-1\right]}
\end{aligned}
$$

$\xi_{m}=\sqrt{m^{2}+\gamma^{2}}$, and the eigenvalues $k_{m n}$ determined by equation $\mathrm{J}_{\xi_{m}}\left(k_{m n} \beta\right) \mathrm{N}_{\xi_{m}}\left(k_{m n} \alpha\right)-\mathrm{J}_{\xi_{m}}\left(k_{m n} \alpha\right) \mathrm{N}_{\xi_{m}}\left(k_{m n} \beta\right)=0$. It is interesting to note that Eq. (11) has formally the same aspect of Eq. (7) and the difference is essentially in the eigenfunction $\widetilde{\Psi}_{m n}\left(r, k_{m n}\right)$, which for this case manifests the influence of the electric field.

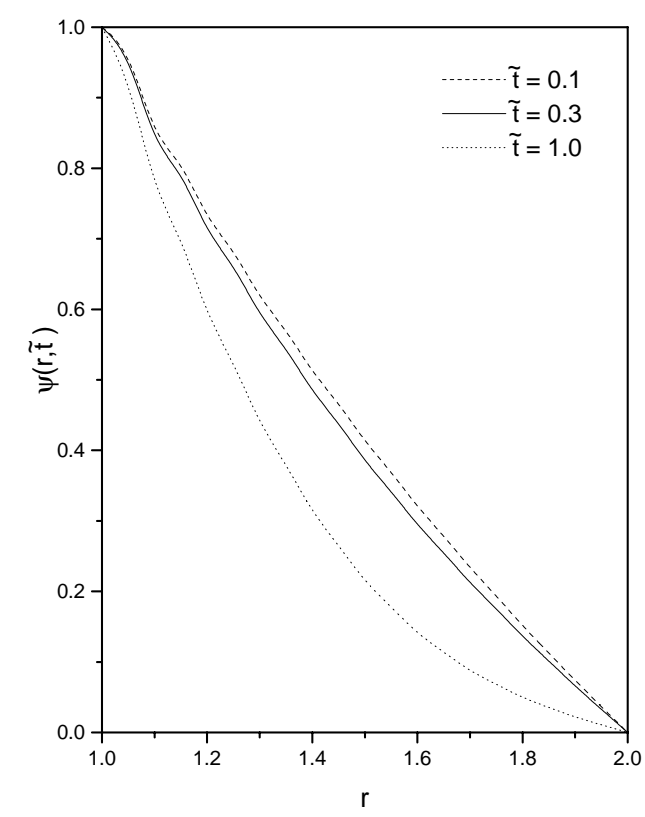

FIG. 2: Behavior of $\psi$ versus $r$ is illustrated in the absence of external field for typical values of $\tilde{t}$ by considering, for simplicity, the initial condition $\psi_{0}(r, \theta)=0, \alpha=1, \beta=2$, and the boundary conditions $\Phi_{a}(\theta, t)=1$ and $\Phi_{b}(\theta, t)=0$.

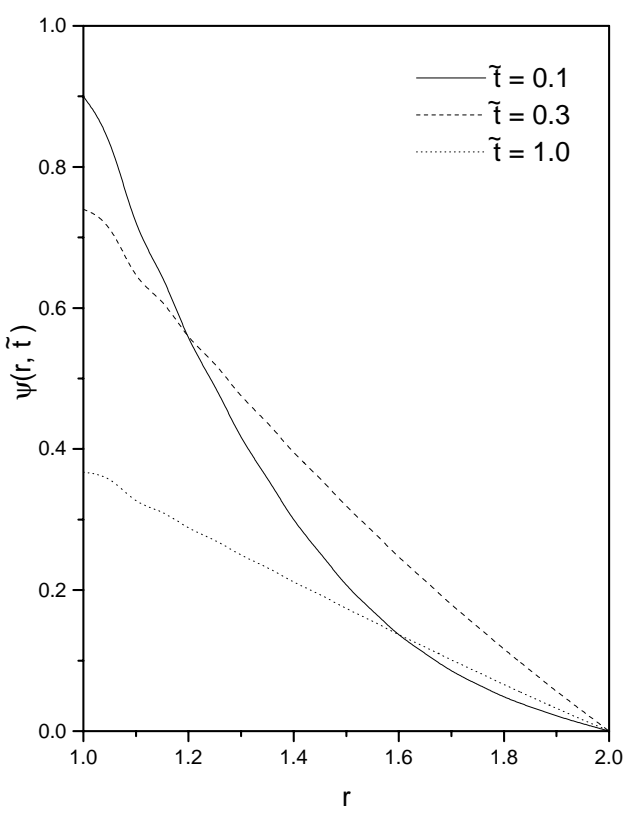

FIG. 3: Behavior of $\psi$ versus $r$ is illustrated in the absence of external field for typical values of $\widetilde{t}$ by considering, for simplicity, the initial condition $\psi_{0}(r, \theta)=0, \alpha=1, \beta=2$, and the boundary conditions $\Phi_{a}(\theta, t)=\Phi_{0} e^{-\delta t}$, with $\Phi_{0}=1$ and $\delta=1 / \tau_{v}$, and $\Phi_{b}(\theta, t)=0$.

In Figs. 2-5, some particular behaviors of the complete tilt angle distribution of Eq. (4) are exhibited for illustrative purposes. In Figs. 2-3, the radial dependence is shown for the initial condition $\psi_{0}(r, \theta)=0$, for specific times and different boundary conditions. In particular, in Fig. 3, we have assumed a time dependent (periodic) easy direction on the inner cylindrical surface. In Fig. $4, \psi(r, \theta, \tilde{t})$ at the position $r=(\alpha+\beta) / 2$ (which coincides with the middle point between the two cylinders) is shown as a function of the field strength $\gamma$ for two limiting situations $t=0$ and $t=\infty$ (stationary situation) to show the effect of the electric field 
in the molecular orientation of the sample. Notice that on the boundaries the easy direction is uniform and planar $\left(\Phi_{a}(\theta, t)=\Phi_{b}(\theta, t)=\pi / 2\right)$, and for $\tilde{t}=0$ the entire sample has this orientation. For large times, the field destroys the planar orientation, as expected if $\varepsilon_{a}>0$, and the orientation at the middle point tends to be the homeotropic one. In Fig. 5, the time dependence of the tilt angle is shown in a particular position $(r=2.5)$ for specific values of the field strength, $\gamma$. As expected, for relatively large times there is a saturation in the value of the tilt angle. The sample tends to a saturated distorted orientation, because we are assuming two different easy direction on the boundaries.

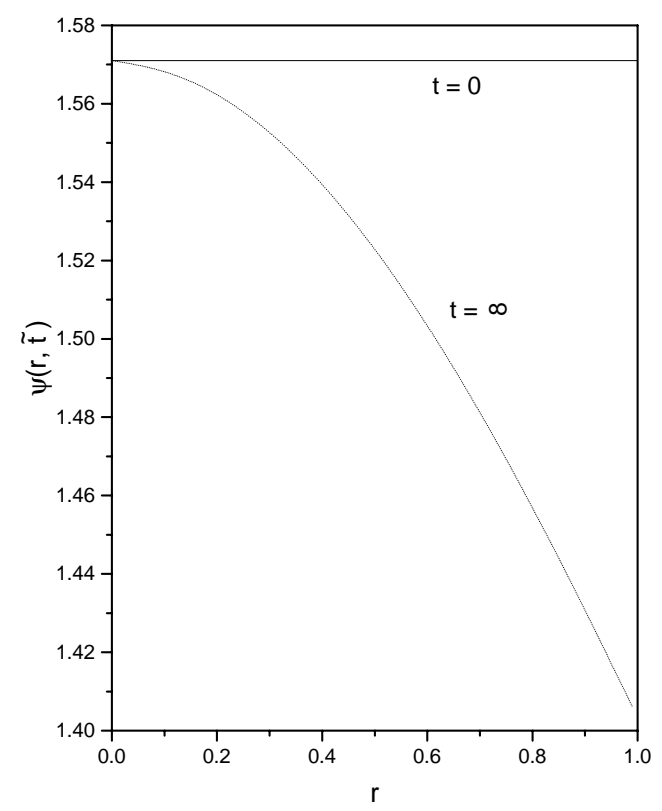

FIG. 4: Behavior of $\psi$ versus $\gamma$ is illustrated for two limiting situations $t=0$ and $t=\infty$ (stationary situation) by considering, for simplicity, $\alpha=1, \beta=2, r=1.5$, the initial condition $\psi_{0}(r, \theta)=\pi / 2$, the boundary condition $\Phi_{a}(\theta, t)=\Phi_{b}(\theta, t)=\pi / 2$.

\section{DISCUSSION AND CONCLUSIONS}

We have worked out the time dependent boundary value problem leading to the determination of the equilibrium director profile in a liquid crystalline sample confined between two concentric cylindrical surfaces. To present an analysis as general as possible for this kind of problem, we have also considered the presence of an external field and a space and time dependence boundary conditions. In principle, this sit- uation could be physically relevant for liquid-crystalline systems confined between surfaces that are inhomogeneous and have a space and time distribution of easy directions. A scenario in which the easy axes change direction continuously, even if difficult to experimentally realize, can be found in

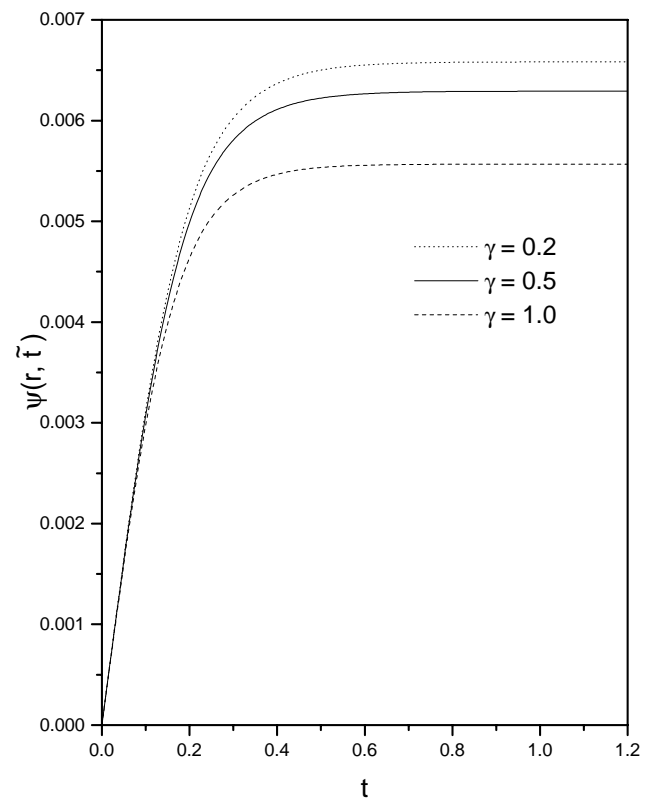

FIG. 5: Behavior of Eq. (4) versus $\tilde{t}$ is illustrated for typical values of $\gamma$ by considering, for simplicity, the initial condition $\psi_{0}(r, \theta)=0$, $r=2.5, \alpha=1 / 3, \beta=4 / 3$, and the boundary conditions $\Phi_{a}(\theta, t)=$ $1 / 100$ and $\Phi_{b}(\theta, t)=1 / 200$.

those systems whose surfaces are covered with photopolymeric films. In these systems, the orientational changes of the photochromic molecules promoted by incident light can lead to remarkable changes in the molecular orientation. The same formalism may also find applications in a diffusive process in the presence of an adsorption phenomenon at the interfaces [20, 21]. For this case, time dependent boundary conditions may be used to represent the dynamical process occurring between the bulk and the surfaces.

\section{ACKNOWLEDGMENTS}

E.K. Lenzi and L.R. Evangelista are especially thankful to the Brazilian Council of Research (CNPq), for continuous funding through a Research Productivity Grant, and to Coordenadoria de Aperfeiçoamento de Pessoal de Nível Superior (CAPES), for funding C. A. R. Yednak. 
[3] D. R. M. Williams and A. Halperin, Phys. Rev. E 48, R2366 (1993).

[4] P. G. de Gennes and J. Prost, The Physics of Liquid Crystals, (Pergamon, Oxford, 1993).

[5] D. R. M. Williams, Phys. Rev. E 50, 1686 (1994).

[6] R. Rosso, E. G. Virga, and S. Kralj, Phys. Rev. E 74, 061703 (2006).

[7] G. Barbero and L. R. Evangelista, An Elementary Course on the Continuum Theory for Nematic Liquid Crystals, (World Scientific, Singapore, 2001).

[8] I. W. Stewart, The Static and Dynamical Continuum Theory of Liquid Crystals, (Taylor and Francis, London, 2004).

[9] C. A. R. Yednak, R. N. Igarashi, E. K. Lenzi, and L. R. Evangelista, Phys. Lett. A 358, 31 (2006).

[10] L. T. Thieghi, R. Barberi, J. J. Bonvent, E. A. Oliveira, J. A. Giacometti, D. T. Balogh, Phys. Rev. E 67, 041701 (2003).

[11] A. Mertelj and M. Copic Phys. Rev. Lett. 81, 5844 (1998); see also references therein.

[12] S. M. Chen, T. C. Hsieh, and R. P. Pan, Phys. Rev. A 43, 2848 (1991).
[13] Z. Zou, N. A. Clark, and T. Carlsson, Phys. Rev. E 49, 3021 (1994).

[14] D. Svensek and S. Zumer, Continuum Mech. Thermodyn. 14, 231 (2002).

[15] C. A. R. Yednak, F. C. M. Freire, E. K. Lenzi, and L. R. Evangelista, Phys. Rev. E 72, 042701 (2005).

[16] S. Chandrasekhar, Liquid Crystals, (Cambridge Univeristy Press, Cambridge, 1977).

[17] M. P. Morse and H. Feshbach, Methods of Theoretical Physics, (McGrawHill, New York, 1953).

[18] F. Bethuel, H. Brezis, B. D. Coleman, and F. Hélein, Arch Rat. Mech. Anal. 118, 149 (1992).

[19] P. J. Barratt and B. R. Duffy, J. Phys. D: Appl. Phys. 29, 1551 (1996).

[20] R. Rossato, M. K. Lenzi, L. R. Evangelista, and E. K. Lenzi, Phys. Rev. E 76, 03212 (2007).

[21] G. Barbero and L. R. Evangelista, Adsorption Phenomena and Anchoring Energy in Nematic Liquid Crystals, (Taylor \& Francis, London, 2006). 